\title{
Model-Invariant Hybrid RANS-LES Computations on Unstructured Meshes
}

\author{
R. Sreekanth* and Stephen L. Woodruff ${ }^{\dagger}$ \\ NASA Langley Research Center, Hampton, Virginia, 23681.
}

\begin{abstract}
Hybrid RANS-LES computations combine the benefits of RANS and LES so that LES is used in regions where the accuracy of RANS deteriorates. The numerous hybrid approaches are limited by the specification of the LES-RANS interface, which can cause nonphysical results such as log-layer mismatch and low shear stress. The hybrid RANS-LES approach based on the concept of model invariance, mitigates these problems, enabling seamless blending of the RANS and LES regions while forming the basis for interpreting the results in the interface region. This hybrid formulation was implemented in the NASA FUN3D unstructured code and computations for flow in a channel at Reynolds number of 3300 (based on the channel half width $h$ and the bulk inflow velocity $u_{\infty}$ ) were carried out. An isotropic stochastic turbulence generator was implemented to generate inflow turbulence. The present approach was able to eliminate the log-layer mismatch and predict the shear stress fairly well. Thus, the model-invariant hybrid formulation coupled with the isotropic turbulence inflow generation serves as a physically meaningful way of performing hybrid RANS-LES computations.
\end{abstract}

\section{Nomenclature}

$\begin{array}{ll}t_{\text {turb }} & \text { Turbulent time scale } \\ \Delta t & \text { Numerical time step } \\ u^{\prime} & \text { Streamwise velocity fluctuation } \\ v^{\prime} & \text { Wall-normal velocity fluctuation } \\ w^{\prime} & \text { Spanwise velocity fluctuation } \\ \delta & \text { Boundary layer thickness } \\ \Delta & \text { Edge length/Filter width } \\ \lambda & \text { Blending parameter } \\ \langle u\rangle & \text { Mean streamwise velocity } \\ \langle v\rangle & \text { Mean wall-normal velocity } \\ \langle w\rangle & \text { Mean spanwise velocity } \\ \mathbf{x} & \text { Position vector } \\ \nu & \text { Laminar viscosity } \\ \omega & \text { Specific dissipation rate } \\ \phi & \text { Primitive variable } \\ \rho_{\infty} & \text { Freestream density } \\ \tilde{\nu} & \text { Eddy viscosity } \\ \tilde{S} & \text { Strain rate } \\ \vec{r} & \text { Distance vector } \\ d & \text { Length scale in SA model } \\ F & \text { Flow quantity that is model invariant } \\ h & \text { Channel half width } \\ k & \text { Turbulent kinetic energy }\end{array}$

*NASA NPP Fellow, Computational AeroSciences Branch. AIAA Member.

${ }^{\dagger}$ Research Scientist, Computational AeroSciences Branch 


\begin{tabular}{|c|c|}
\hline$L$ & Length of channel \\
\hline$p$ & Static pressure \\
\hline$q$ & Vector of primitive variables \\
\hline$s$ & Model sensitivity \\
\hline$u_{\infty}$ & Centerline inflow velocity/Inflow Mach number \\
\hline$u_{\tau}$ & Reference frictional velocity at inflow \\
\hline$x, y, z$ & Cartesian coordinate directions \\
\hline$y^{+}$ & Distance of a grid point from nearest wall in wall units \\
\hline$y_{w a l l}^{+}$ & Distance of the first grid point from the wall in wall units \\
\hline \multicolumn{2}{|c|}{ Subscripts } \\
\hline$i, j$ & Node indices \\
\hline$L$ & Left state \\
\hline$l, m$ & Tensor indices \\
\hline$R$ & Right state \\
\hline \multicolumn{2}{|c|}{ Abbreviations } \\
\hline CFD & Computational fluid Dynamics \\
\hline DES & Detached eddy simulation \\
\hline FUN3D & Fully unstructured Navier Stokes three-dimensional solver \\
\hline LES & Large eddy simulation \\
\hline MI & Model invariant hybrid RANS-LES \\
\hline RANS & Reynolds averaged Navier Stokes \\
\hline $\mathrm{SA}$ & Spalart-Allmaras \\
\hline SST & Shear-stress transport \\
\hline \multicolumn{2}{|c|}{ Constants } \\
\hline$\beta$ & Constant in $k-\omega$ model \\
\hline$\beta^{*}$ & Constant in $k-\omega$ model \\
\hline$\gamma$ & Constant in $k-\omega$ model \\
\hline$\kappa$ & Karman constant \\
\hline$\phi_{M}$ & Constant in MUSCL Scheme \\
\hline$\sigma$ & Constant in $k-\omega$ model \\
\hline$\sigma_{\omega}$ & Constant in $k-\omega$ model \\
\hline$\sigma_{k}$ & Constant in $k-\omega$ model \\
\hline$c_{b 1}$ & Constant in SA Model \\
\hline$c_{b 2}$ & Constant in SA Model \\
\hline$c_{w}$ & Constant in SA Model \\
\hline$f_{12}$ & Constant in SA Model \\
\hline$f_{w}$ & Constant in SA Model \\
\hline
\end{tabular}

\section{Introduction}

A fully wall-resolved large-eddy simulation (LES) is computationally prohibitive for all but the simplest wall-bounded flows. Any fully resolved LES must capture the turbulent scales in the flow field that contain most of the turbulent kinetic energy and Reynolds stresses. Thus, the grid spacing in the near-wall regions has to be nearly as fine as that employed in a direct-numerical simulation. Reynolds-Averaged Navier-Stokes (RANS) computations, on the other hand, are less computationally intensive but fail severely in complex flows involving separation and reattachment. Detached Eddy Simulation (DES) was introduced by Spalart et al. ${ }^{1}$ as a way of blending the two approaches to alleviate the pitfalls in each, while utilizing the benefits of both approaches. Over the years, numerous methods of performing DES and hybrid methods involving blending LES and RANS have been developed. Most of these approaches tend to be very sensitive to the specification of the transition region from RANS to LES. Nikitin et al. ${ }^{2}$ showed that even for simple flow configurations such as a channel flow, placing the LES-RANS interface region in the log-layer causes nonphysical results such as the log-layer mismatch. The log-layer mismatch results in overpredicted mean velocities toward the center of the channel and poor shear stress prediction over the entire channel. Kawai and Larson ${ }^{3}$ showed that wall-modeled LES has similar sensitivity to the placement of the wall modeling region. 
A number of techniques have been proposed to alleviate this problem, such as: addition of turbulent fluctuations to the LES-RANS interface region (Keating et al., ${ }^{4}$ Shur et al. ${ }^{5}$ ), refining the grid to resolve the outer part of the boundary layer (Kawai and Larson $^{3}$ ), providing better correlations for the blending parameter (Shur et al. ${ }^{6}$ ) as well as modeling the commutation error (Wallin et al. ${ }^{7}$ ). While many of these approaches have achieved some success, development of a generalized method for the arbitrary placement of the RANS-LES interface remains an open challenge.

The model-invariant hybrid method developed by Woodruff ${ }^{8}$ addresses this problem by enabling a seamless blending of flow variables and establishing a basis for interpreting the results in the LES-RANS interface region. Typically in hybrid RANS-LES methods, a blending parameter is used to switch between the LES and RANS regions of the flow field. A continuously-blended hybrid model is defined by the blending parameter $\lambda$ such that $\lambda$ varies continuously from 0 to 1 . The hybrid model exhibits LES behavior when $\lambda=1$ and RANS behavior when $\lambda=0$; any value of $\lambda$ in between $(0<\lambda<1)$ involves a mix of both LES and RANS behavior.

The central idea of the model-invariant hybrid approach is that physical quantities should be independent of the blending parameter and thus are model invariant. A physically meaningful hybrid computation, independent of the blending parameter, has to be based on model invariants. ${ }^{8}$ The mean velocity in the flow field may be considered a model invariant, since the mean velocity at a point should be the same irrespective of whether the hybrid model operates in the RANS or LES modes. Similarly, the total turbulent kinetic energy is another model invariant. In a model-invariant hybrid computation, the total turbulent kinetic energy, when expressed as the sum of the resolved and modeled kinetic energy, does not change at the RANS-LES interface due to variation in the blending parameter. The use of model invariance allows comparison of flow quantities computed with different blends of RANS and LES to their RANS equivalents. A model invariant $F$ has to satisfy the constraint $(\partial F / \partial \lambda)=0$.

Tests on the model invariant hybrid RANS-LES were carried out for isotropic decaying turbulence, channel flow, and a periodic hill in Refs. [8-10], respectively. In the present work, DES using the Spalart-Allmaras ${ }^{1}$ and Strelets ${ }^{11}$ approaches for flow in a channel were carried out using NASA's FUN3D unstructured code to demonstrate the log-law mismatch. Subsequently, the model invariant hybrid formulation was implemented in FUN3D and simulations were carried out. An isotropic turbulence generator was implemented to add fluctuations at the inflow plane, so that there are sufficient fluctuations in the flow field for the LES to resolve when the DES/hybrid LES-RANS model switches from RANS to the LES mode. The problem simulated, the computation parameters used, equations solved, the new features added to FUN3D, the implementation of model invariant approach, grid generation procedure and analysis of the results are presented in the subsequent sections of this paper.

\section{Problem Description}

The canonical problem of fully-developed turbulent flow in a channel has been studied extensively in the turbulence community to enable a better understanding of the physics of wall-bounded turbulent flows. The geometrical simplicity of the problem is attractive in both experimental and numerical research. A schematic diagram of the problem is shown in Figure 1. A vast amount of experimental research (Nikuradse, ${ }^{12}$ Reichardt, ${ }^{13}$ Laufer,${ }^{14}$ Comte-Bellot ${ }^{15}$ ) and numerical literature (Kim et al., ${ }^{16}$ Moin et al., ${ }^{17}$ Nikitin et al. ${ }^{2}$ ) exists on this problem. The presence of a large database of these results has been vital to the development of turbulence models, turbulence generators, wall models for RANS/LES, subgrid models and so on. In the present work, channel flow for a Reynolds number of 3300 based on the half width $h$ of the channel and the centerline inflow velocity $u_{\infty}$ was simulated. The Reynolds number $R e_{\tau}$ based on the friction velocity $u_{\tau}$ at the inflow is 180 . The physical domain consists of walls at the top and bottom, inflow on the left boundary and outflow on the right boundary. The governing equations used in the present work being the compressible Navier-Stokes equations, the following boundary conditions are imposed: No-slip and adiabatic boundary conditions were specified on the top and bottom walls ( $y$ direction) of the channel while periodic boundary conditions were specified on the sides in the spanwise $(z)$ direction. At the outflow boundary, the static pressure was prescribed. The ratio of the static pressure to the reference pressure was 1.0. The flow is driven by a pressure gradient imposed at each control volume as a body force. The pressure gradient was computed as

$$
\frac{\Delta p}{L}=\rho_{\infty} u_{\tau}^{2}
$$




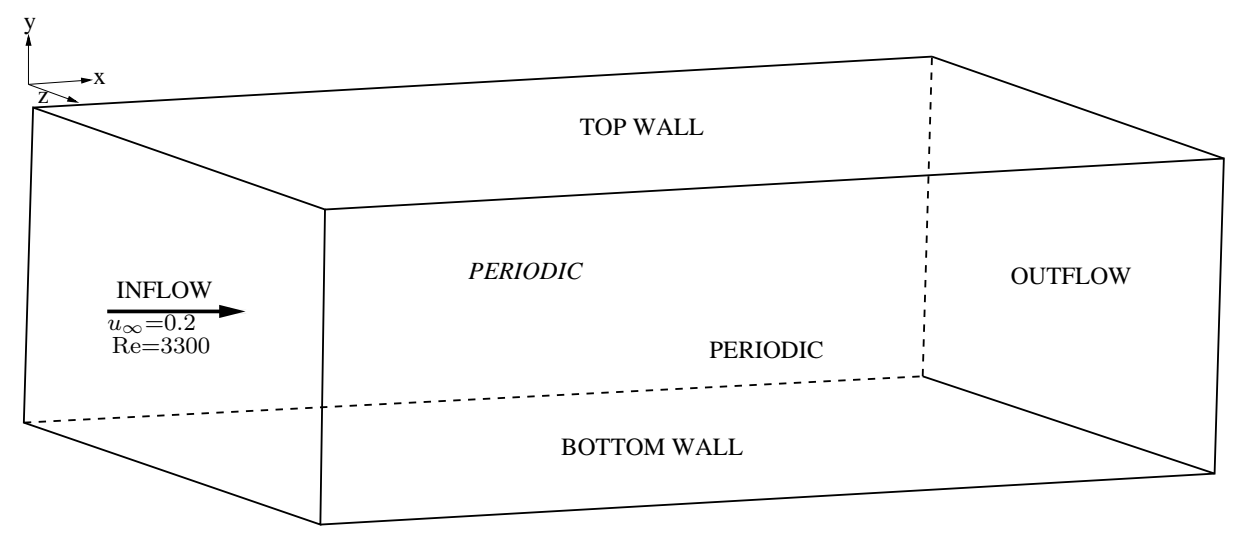

Figure 1. Schematic diagram of the channel flow problem.

where $\rho_{\infty}$ is the freestream density, $L$ is the streamwise length of the channel and $u_{\tau}$ is the frictional velocity. The inflow conditions consist of two components, the mean flow and synthetic fluctuations, which are superimposed on the mean flow. The mean velocity profile was obtained from the DNS data of Kim et al. ${ }^{16}$ Figure 2 shows the mean velocity profile prescribed at the inflow boundary.

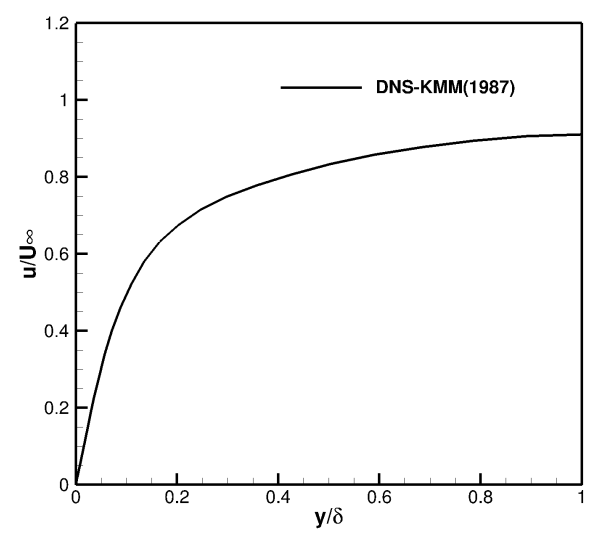

Figure 2. Mean inflow velocity profile.

The synthetic turbulence imposed is generated using the method described in section (VI). The centerline inflow velocity is 0.2 , and the freestream reference temperature was $540^{\circ} \mathrm{R}$. A stagnation pressure ratio of 1.02828 with respect to the reference pressure were specified at the inflow to ensure that the ratio of the static pressure to the reference was 1.0. The nondimensional time step $\Delta t$ used in the simulation is $4.392 \times 10^{-4} h / u_{\tau}$. The size of the computational domain in the streamwise $(x)$ direction is $2 \pi h, 2 h$ in wall normal direction, and $4 \pi h / 3$ in the spanwise direction. The domain size in the streamwise direction is shorter than that of some simulations in the literature ${ }^{2,16}$ this was done to reduce the computational time. The shortened domain size has no effect on the simulations since the two-point correlation and energy spectra were shown to go to zero at these domain lengths in Ref. 16.

\section{Governing Equations}

The governing equations used in the present work are the compressible Navier-Stokes equations with the Spalart-Allmaras (SA) based DES (Spalart et al. ${ }^{1}$ ) and the DES model of Strelets. ${ }^{11}$ 


\section{Spalart-Allmaras DES(SA-DES)}

The Spalart-Allmaras DES model, Spalart et al., ${ }^{1}$ is given by

$$
\frac{\partial \tilde{\nu}}{\partial t}+u_{m} \frac{\partial \tilde{\nu}}{\partial x_{m}}=c_{b 1}\left(1-f_{12}\right) \tilde{S} \tilde{\nu}=\left[c_{w 1} f_{w}-\frac{c_{b 1}}{\kappa^{2}} f_{12}\right]\left(\frac{\tilde{\nu}}{d}\right)^{2}+\frac{1}{\sigma}\left[\frac{\partial}{\partial x_{m}}\left((\nu+\tilde{\nu}) \frac{\partial}{\partial x_{m}}\right)+c_{b 2} \frac{\partial \tilde{\nu}}{\partial x_{l}} \frac{\partial \tilde{\nu}}{\partial x_{l}}\right] .
$$

The parameter $d$ in the destruction term is computed as

$$
\tilde{d}=\min \left(d, C_{D E S} \Delta\right)
$$

where $\Delta$ is the maximum edge length in the dual volume. The value of $d$ is chosen such that the SA model mimics the Smagorinsky LES subgrid model in regions where the resolution is sufficiently fine and behaves as a default SA RANS model in regions where grid resolutions are insufficient, typically found in the attached regions on the flow. The value of $C_{D E S}$ used in the present work is 0.65 while the remaining constants have their usual values (Spalart et al. ${ }^{1}$ ).

\section{DES of Strelets}

The DES model of Strelets ${ }^{11}$ is based on the Menter two-equation shear-stress transport (SST) model. ${ }^{18}$

$$
\begin{gathered}
\frac{\partial k}{\partial t}+u_{m} \frac{\partial k}{\partial x}=P-D+\frac{\partial}{\partial x_{m}}\left[\left(\mu+\sigma_{k} \mu_{t}\right) \frac{\partial k}{\partial x_{m}}\right] \\
\frac{\partial \omega}{\partial t}+u_{m} \frac{\partial \omega}{\partial x}=\frac{\gamma}{\mu_{t}} P-\beta \omega^{2}+\frac{\partial}{\partial x_{m}}\left[\left(\mu+\sigma_{\omega} \mu_{t}\right) \frac{\partial \omega}{\partial x_{m}}\right] .
\end{gathered}
$$

Here $\mathrm{D}$ is the dissipation term and is given by $D=k^{3 / 2} / l_{h}, \mathrm{P}$ is the production term and is given by $P=\mu_{t} \tilde{S}_{l m} \tilde{S}_{l m}$ and $\tilde{S}_{l m}$ is the symmetric portion of the velocity gradient tensor. The length scale $l_{H}$ is defined as

$$
l_{H}=\min \left(k^{1 / 2} /\left(\beta^{*} \omega\right), C_{D E S} \Delta\right) .
$$

The choice of $l_{H}$ makes the model mimic a Smagorinsky type subgrid scale model under equilibrium conditions while providing the typical $k-\omega$ RANS model behavior otherwise. The value of $C_{D E S}$ used here is 0.65 while the remaining constants have their usual values. ${ }^{11}$

\section{Model-Invariant Hybrid RANS-LES Formulation(MI)}

The model-invariant formulation developed by Woodruff ${ }^{8}$ is based on the continuous modeling approach of Speziale. ${ }^{19}$ The form of the approach employed here adapts the DES model of Strelets ${ }^{11}$ and is based on the same equations (4 and 5). The approach makes a smooth transition from the RANS to the LES regions by gradually changing the blending parameter from zero to one while at the same time removing the artifacts introduced into the flow physics due to the variation of the blending parameter. The continuous, smooth, blending between the RANS and LES regions is achieved by blending the dissipation terms in the LES and RANS modes of the model. The dissipation term $\mathrm{D}$ is thus given by

$$
D=(1-\lambda) \beta^{*} \omega k+\lambda k^{3 / 2} /\left(C_{D E S} \Delta\right)
$$

where $\lambda$ is the blending parameter, which varies between 0 and 1 , with the standard values of constants used in the $k-\omega$ model.

To introduce the model-invariant hybrid RANS-LES approach into the governing equations, the constant blending parameter $\lambda$ is replaced by a spatio-temporal function $\lambda\left(t^{\prime}, x^{\prime}\right)$. As a result of using a variable $\lambda$, the governing equations need to be modified to account for the change of flow variables due to the spatio-temporal variation in the blending parameter.

The constant blending-parameter and variable blending-parameter scenarios are connected by a simple coordinate transformation of the independent variables in the governing equations. If $\left(t^{\prime}, x^{\prime}, \xi\right)$ are the coordinates of the constant- $\lambda$ case, and $(t, x, s)$ are the coordinates of the variable- $\lambda$ scenario, the two coordinates 
systems can be related by $t^{\prime}=t, x^{\prime}=x$ and $s=\xi-\lambda\left(t^{\prime}, x^{\prime}\right)$. Introduction of the new coordinate system results in the modification of the spatial and temporal derivatives in all the governing equations. The temporal derivatives are transformed as

$$
\frac{\partial}{\partial t^{\prime}}=\frac{\partial}{\partial t}-\frac{\partial \lambda}{\partial t} \frac{\partial}{\partial s}
$$

and the spatial derivatives as

$$
\nabla^{\prime}=\nabla-(\nabla \lambda) \frac{\partial}{\partial s}
$$

The application of the coordinate transformation results in the addition of several source terms in the Navier-Stokes and $k-\omega$ equations. The derivatives of the flow variables with respect to the model sensitivity parameter $s$ are evaluated according to simple but effective approximations as detailed in Ref. 9 .

\section{The Numerical Method}

FUN3D is a node-based, finite-volume flow solver..$^{20,21}$ The model-invariant formulation of the governing equations described in the previous section was implemented in the NASA FUN3D CFD code. A detailed description of FUN3D and its use can be found in Biedron et al. ${ }^{22}$ The compressible Navier-Stokes equations along with the turbulence model equations were nondimensionalized and solved in a segregated manner, i.e., the governing equations are solved one by one over the entire domain. The convective fluxes in FUN3D are computed using a novel low dissipation version the of Roe's scheme ${ }^{23}$ while flux reconstruction is done using Van Leer's MUSCL procedure. Figure 3 shows the stencil for the MUSCL scheme with an unstructured dual volume discretization, where $i j$ is an edge connecting two nodes $i$ and $j$. The point $P$ is the midpoint of the face center of the dual volume. The left and right states at the interface $P$ are given by $q_{i j}^{L}, q_{i j}^{R}$,

$$
\begin{aligned}
q_{i j}^{L} & =q_{i}+r_{(i, i j)} \frac{\phi_{M}}{4}\left[\left(1-\kappa_{M}\right) \Delta q_{i}+\left(1+\kappa_{M}\right) \Delta q_{i}\right] \\
q_{i j}^{R} & =q_{j}+r_{(j, i j)} \frac{\phi_{M}}{4}\left[\left(1-\kappa_{M}\right) \Delta q_{j}+\left(1+\kappa_{M}\right) \Delta q_{j}\right]
\end{aligned}
$$

where $q_{i}$ and $q_{j}$ are the vectors of the primitive variables, and $\Delta q_{i}, \Delta q_{j}$ are their gradients at the nodes $i$ and $j . r_{(i, i j)}, r_{(j, i j)}$ are the positional vectors from the nodes $i$ and $j$ to the point $P$, along the edge $i j$, respectively. The parameter $\phi_{M}$ varies between 0 and 1 and is used to control the order of reconstruction, $\phi_{M}=0$ gives first order reconstruction, while $\phi_{M}=1$ results in higher-order reconstruction. A value of $\phi_{M}=1$ was used in the present work to obtain second-order accuracy. The parameter $\kappa_{M}$ can be varied between 0 and $1, \kappa_{M}=0$ results in an upwind biased (Fromm's) scheme, while $\kappa_{M}=1$ results in an unstable central difference scheme. In the present work, $\kappa_{M}$ was set to 0.99 to mimic central difference behavior to

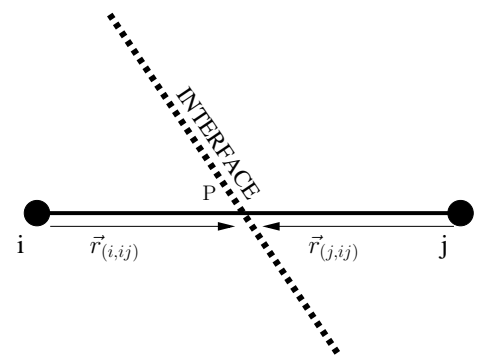

Figure 3. Dual volume stencil for the MUSCL scheme.

minimize dissipation. The gradients were evaluated with second-order least-squares reconstruction. Time integration was carried out using a second order, point-implicit backward-difference scheme. At each time step, the linear system of equations is approximately solved with a multicolor point-implicit procedure and about 20 subiterations were used per time step. 


\section{V.A. Grid Generation}

The grids used in the present work are described in Table 1. The simulations were first carried out on two hexahedral grids and finally an equivalent anisotropic tetrahedral grid corresponding to the finest hexahedral grid was used.

Table 1. Grids used in the present work.

\begin{tabular}{|c|c|c|c|c|c|c|}
\hline Grid & Type & Wall-Normal & Streamwise & Spanwise & $y_{\text {wall }}^{+}$ & Control volumes \\
\hline 1 & Hexhederal & 100 & 80 & 80 & 1 & 640000 \\
2 & Tetrahedral & 100 & 80 & 80 & 1 & 602000 \\
\hline
\end{tabular}

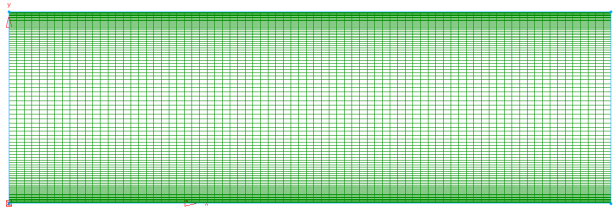

(a) Hexahedral grid.

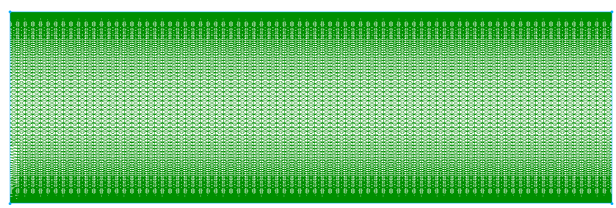

(b) Anisotropic tetrahedral grid.

Figure 4. Finest hexahedral and tetrahedral grids used in present work.

The first hexahedral grid had 80 points in all directions and the first grid point away from the wall was fixed at $y^{+}=4$. This resulted in poor prediction of the resolved shear stress with the SA-DES model and hence the grid was refined in the wall normal direction so that the first grid point away from the wall was at $y^{+}=1$. The final hexahedral grid used shown in Figure 4(a) had a total of 640000 grid points. The wall normal $(y)$ direction had 100 points, while 80 points were retained in the streamwise $(x)$ direction and spanwise $(z)$ directions. A uniform grid spacing was used in the $x$ and $z$ directions $(\delta x=0.078$ and $\delta z=0.052)$. The growth rate of the mesh in the wall normal direction was 1.05 to ensure that there were 40 points in the boundary layer. This meshing strategy was chosen in order to capture the eddies near the wall and in the log layer which triggers turbulence, by enabling the LES mode of the DES/hybrid model.

The anisotropic tetrahedral mesh was generated with a similar distribution of grid points as the finest hexahedral mesh. A growth rate of 1.05 with 40 full anisotropic tetrahedral layers in the near wall region was specified. The final grid had about 3.5 million tetrahedral elements with 602000 nodes. The use of tetrahedral elements is justified, since FUN3D, being an unstructured node-based, dual-volume solver, is much faster on tetrahedral meshes than on hexahedral meshes with the same number of nodes. This is because the number of internal computations and memory accesses in FUN3D for a dual volume mesh, composed of hexahedrons, is much higher than that of tetrahedrons.

\section{Spatial Averaging}

A new capability for spatial averaging on unstructured grids was implemented in FUN3D by using a combination of the ray-tetrahedron-intersection and point-in-tetrahedron algorithm. The implementation of the spatial averaging procedure is shown in Figure 5. Here $y$ is considered as the wall normal direction, while $x$ and $z$ are the (homogeneous) streamwise and spanwise directions. The CFD solver cannot traverse unstructured grids in an ordered fashion due to the unordered indexing of the elements. Therefore, performing spatial averaging in such grids is a challenge. For simple geometries such as channels, ducts, or pipes, spatial averaging can be accomplished by merely specifying the spanwise and streamwise directions of the flow domain and the number of planes for averaging in each direction. The extrema of the averaging domain is specified using a bounding box where $P 1$ and $P 2$ (see Figure 5(b)) are the extrema in the wall-normal direction. The whole computational domain is triangulated, and locations of $P 1$ and $P 2$ are found with a parallel point-in-triangle algorithm on the boundary triangular elements using Plucker coordinates. Subsequently, all the elements that intersect the wall normal line $P 1 P 2$ are obtained using a ray-tetrahedron-intersection algorithm (see Figure 5(a)) with the bounding box as limits. Thus, with the wall-normal, streamwise and 
spanwise coordinate information known, a corresponding structured grid is generated. The cell centers of the structured grid are then computed.

Subsequently, the structured grid of the averaging domain is traversed in the spanwise and streamwise directions while simultaneously a search is performed for the set of wall-normal cell centers in the flow domain by using a point-in-tetrahedron algorithm. This is illustrated in Figure 5(c), which shows the immersion of a structured grid inside the original unstructured tetrahedral mesh of the computational domain. When a point in a tetrahedron is found, the value of the flow variable or required output quantity at the point is computed by interpolating from the nearest node or edge. If the point is not found inside the tetrahedron, the nearest value from the closest edge or node is used. The search algorithm is carried out in parallel, making it an extremely scalable operation. For complex geometries with one or more homogeneous directions, the structured grid for averaging can be generated using a commercial grid generator such as Pointwise ${ }^{\circledR}$ and inputted to FUN3D in the PLOT3D format. The algorithm is flexible, performing spatial averaging only in the spanwise direction at specific streamwise locations or vice versa. For the channel flow case simulated in the present work, spatial averaging is performed in both the spanwise and streamwise directions as depicted in Figure 5(b). The arrows show the sweeping in the streamwise $(x)$ direction and spanwise $(z)$ direction.

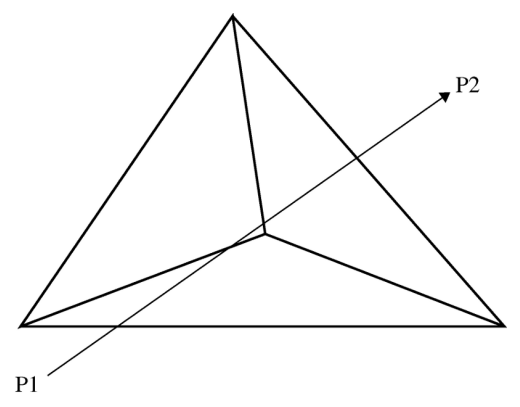

(a) Ray-tetrehdron interesection

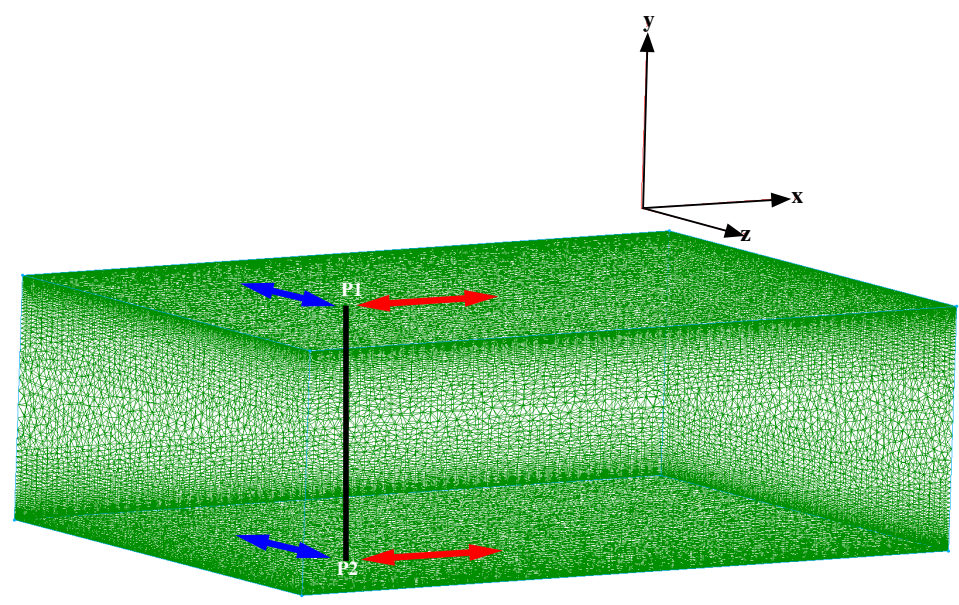

(b) Spatial average sweeping

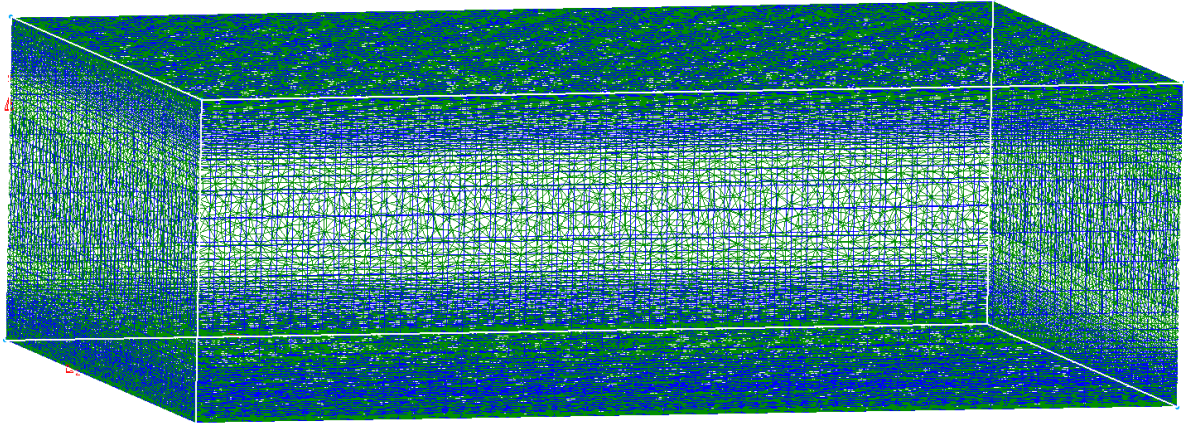

(c) Structured grid for the averaging domain, immersed in the unstructured grid of the flow domain

Figure 5. Implementations of Spatial Averaging Procedure in FUN3D. 


\section{Inflow Turbulence Generation}

In the present work, the stochastic isotropic turbulence generator of Davidson ${ }^{24}$ is used to generate turbulence at the inflow boundary. The velocity at the inflow plane is given by

$$
U_{l}(x, t)=\left\langle U_{l}\right\rangle+v_{l}^{\prime}(x, t)
$$

where $\left\langle U_{l}\right\rangle$ is the mean velocity and $v^{\prime}(x, t)$ is the vector of the velocity fluctuations. The isotropic fluctuations, $u_{l}^{\prime}(x, t)$ are computed as a sum of weighted spatio-temporal Fourier modes.

$$
u_{l}^{\prime}(x, t)=2 \sum_{n=1}^{N} \sigma_{l}^{n} q^{n} \cos \left(k_{l}^{n} d_{l}^{n} \mathbf{x}_{l}+\psi^{n}\right) .
$$

Here $N$ is the number of modes, $q^{n}$ is amplitude of the $n^{t h}$ mode defined by a prescribed energy spectrum, $k^{n}$ is the wave number corresponding to the mode $n, d^{n}$ is random unit vector of direction uniformly distributed over a sphere, $\sigma^{n}$ is the unit vector normal to $d^{n}$, and $\psi^{n}$ is the phase of the mode $n$. The amplitudes of the Fourier modes $q^{n}$ in Eq. (13) is computed as

$$
q^{n}=\sqrt{E\left(k^{n}\right) u_{r m s}^{2} \Delta k^{n}}
$$

where $E\left(k^{n}\right)$ is the energy spectrum of the turbulent kinetic energy given by

$$
E(k)=\frac{\alpha}{k_{e}} \frac{\left(k^{n} / k_{e}\right)^{4}}{\left[1+\left(k^{n} / k_{e}\right)^{2}\right]^{17 / 6}} f_{\text {eta }}
$$

where $\alpha=1.45$ is a constant and $f_{\text {eta }}$ is the dissipation spectral function ( Davidson ${ }^{24}$ ). A total of $125-350$ modes with a linear distribution of wave numbers was used to generate the fluctuations. A constant integral length scale between $0.06 h-0.1 h$ was used. The rms value of the fluctuations was fixed at $20 \%$ of the centerline inflow velocity. Time filtering for the isotropic fluctuations was introduced using an asymmetric time filter.

$$
v_{l}^{\prime}\left(x, t^{m}\right)=a v_{l}^{\prime}\left(x, t^{m-1}\right)+b u_{l}^{\prime}\left(x, t^{m}\right)
$$

where $m$ is the time step number, $a=\exp \left(\Delta t / t_{\text {turb }}\right)$ and $b=1-a^{2}, t_{\text {turb }}$ is the turbulent time scale. The turbulent time scale is computed as

$$
t_{\text {turb }}=\frac{1}{t_{f a c}}\left(0.05 h / u_{r m s}\right)
$$

where $t_{f a c}$ is a scaling factor, which varies from 0 to 1 . The time scale of the turbulent fluctuations had a vital effect in generating sustainable fluctuations in the domain. The value of $t_{f a c}$ used in the present work was $0.3-0.8$. The ratio of the turbulent time scale to the numerical time step $\left(t_{\text {turb }} / \Delta t\right)$ was found to be a critical parameter for inflow generation in the present work. A ratio of 1.5-10 was used in the present work. As a general rule of thumb, using a turbulent time scale that is much larger than the numerical time step ensured high fidelity fluctuations are generated that trigger the equations to resolve turbulence and produce fairly accurate resolved shear stress. Slices of the inflow plane with the three velocity components are shown in Figure 6. The quality of turbulence produced depended on the length scale $l_{t}$, the number of modes $N$, the time scale of the fluctuations $t_{\text {turb }}$ and the rms of the fluctuations. After numerous tests, it was observed that only the right blend of these parameters could produce sufficient fluctuations that trigger the equations to resolve the turbulence. 


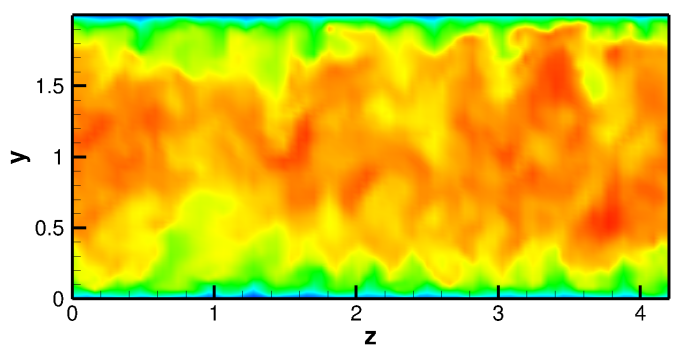

(a) u-velocity

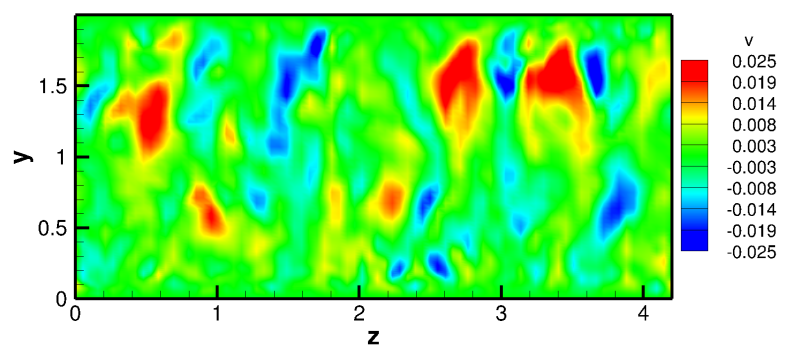

(b) v-velocity

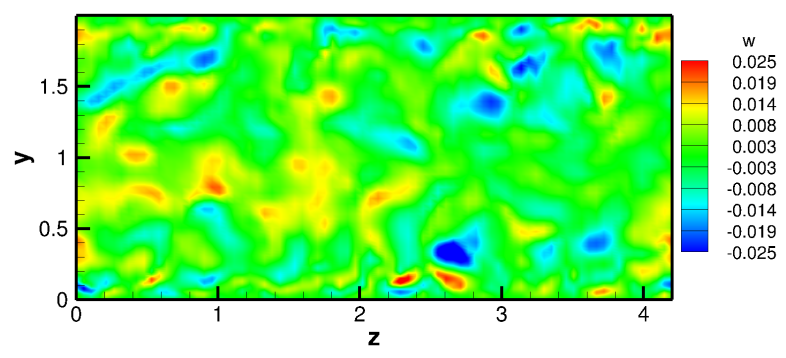

(c) w-velocity

Figure 6. Velocity components at inlet plane with isotropic stochastic turbulent fluctuations.

\section{Results and Discussion}

\section{VII.A. Results using SA-DES}

In this section, results for the channel flow case using the existing SA-DES model in FUN3D are presented. Figure 7 shows the spatial and temporal-averaged mean streamwise velocity and resolved shear stress profiles. The results shown in Figure 7 were obtained on the finest hexahedral grid described in Section 1. (Grid No. 2 in Table 1). The mean velocity and shear stress are in good agreement with the DNS results of Kim et al. ${ }^{16}$ For this simulation, 125 modes, a length scale of 0.06 and a turbulent time scale of $1.56 \Delta t$ were used as inputs to the turbulence generator. 


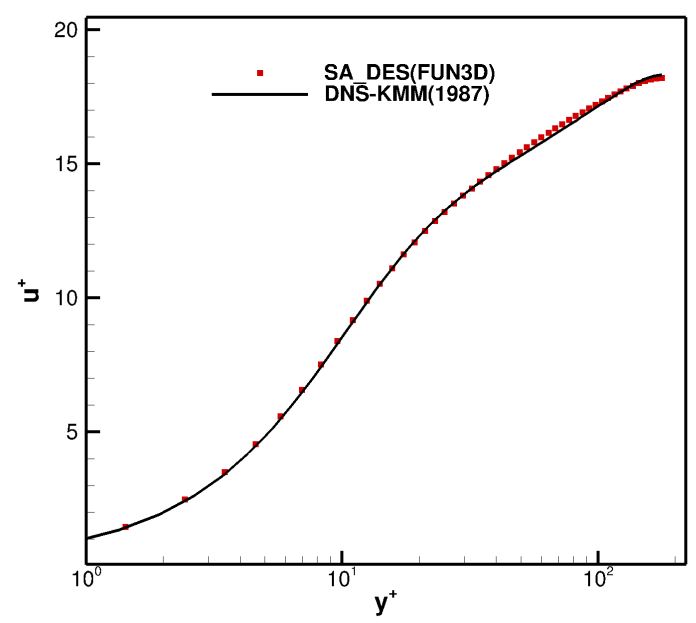

(a) Mean streamwise velocity $\langle u\rangle$

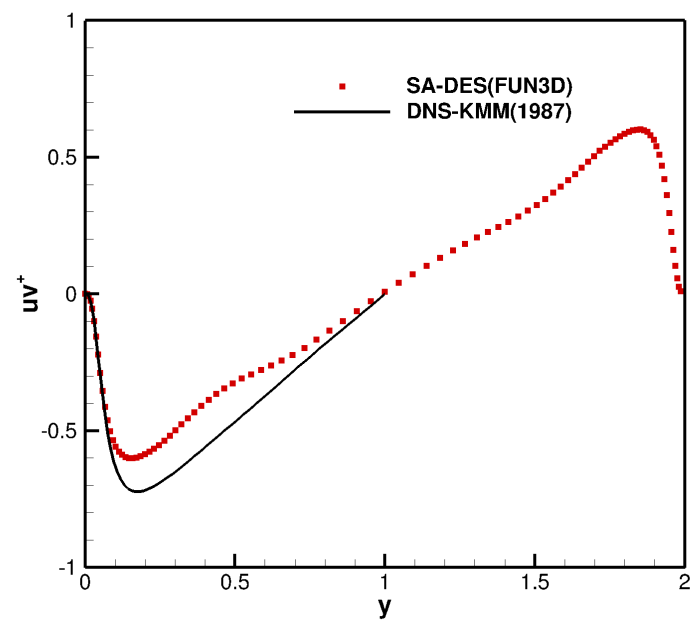

(b) Resolved shear stress $\langle u v\rangle$

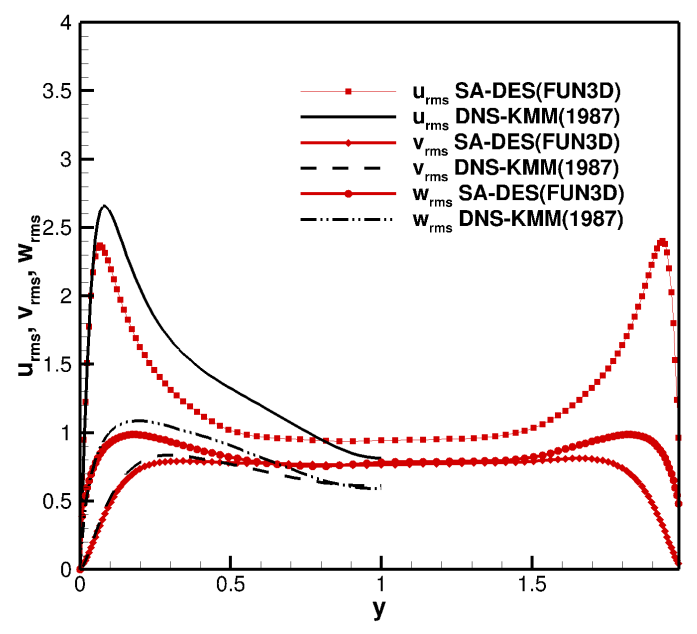

(c) RMS of velocities

Figure 7. SA-DES results. 


\section{VII.A.1. Streamwise variations}

The streamwise variation of the turbulence quantities are shown in Figure 8. The flow is underdeveloped before $1 h$ and becomes reasonably fully developed beyond $1 h$. There is still marginal decay of the fluctuations beyond $1 h$ due to the numerical dissipation. The mean velocity and resolved shear stress are in good agreement with the DNS data at streamwise locations beyond $1 \mathrm{~h}$. The SA-DES simulations were primarily used to validate the operative capability of FUN3D after incorporation of all the new features such as inflow generation, spatial averaging and to lay down a common platform for evaluation of all the DES and hybrid-RANS-LES computations in the present work.

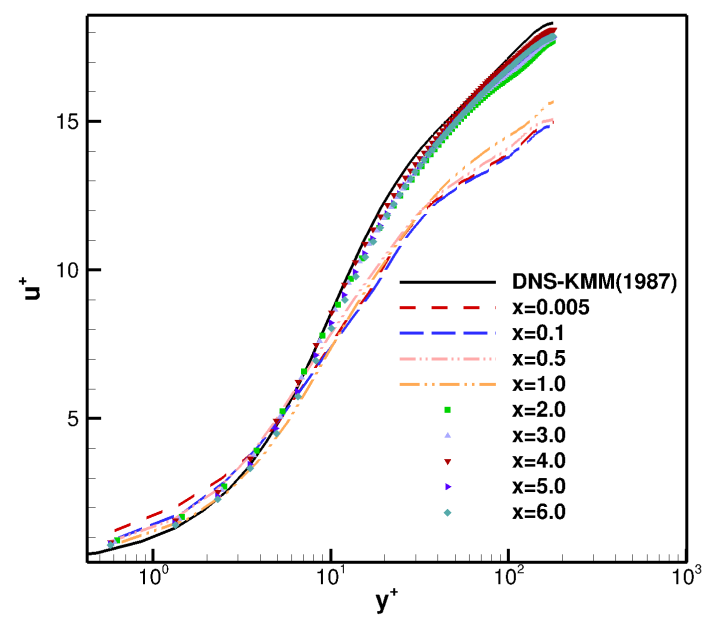

(a) Mean streamwise velocity $\langle u\rangle$

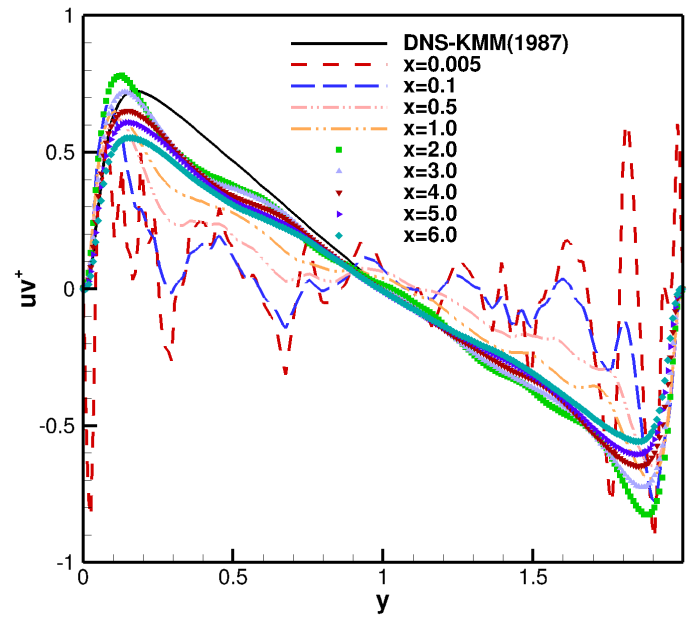

(b) Resolved shear stress $\langle u v\rangle$

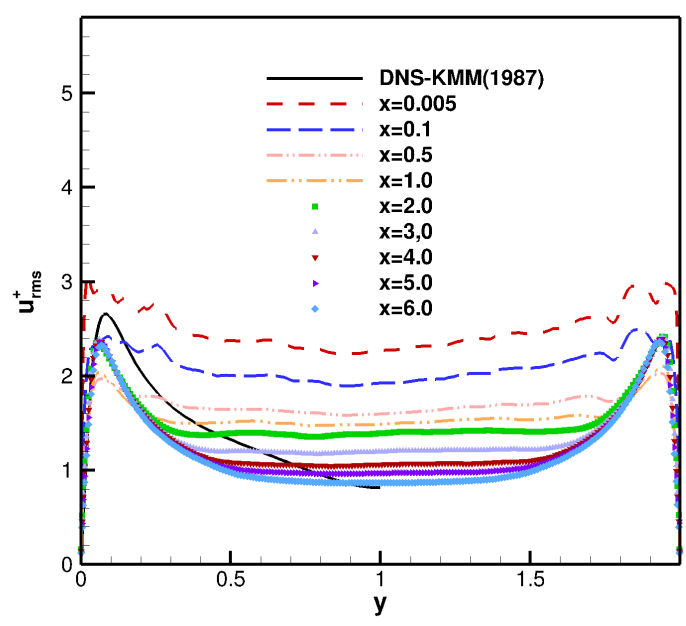

(c) RMS of streamwise velocity $u_{r m s}$

Figure 8. SA-DES streamwise results. 


\section{VII.A.2. $C_{p}$ and $C_{f}$ Fluctuations at the Wall}

The comparison of channel flow results obtained using incompressible and compressible flow codes requires some careful attention. Compressible flow simulations, particularly those carried out at low Mach numbers and resemble internal flow configurations, are susceptible to acoustic fluctuations in the domain. The contours of the instantaneous values of $C_{p}$ and $C_{f}$ on the bottom wall $(y=0)$ of the channel are shown in Figure 9.

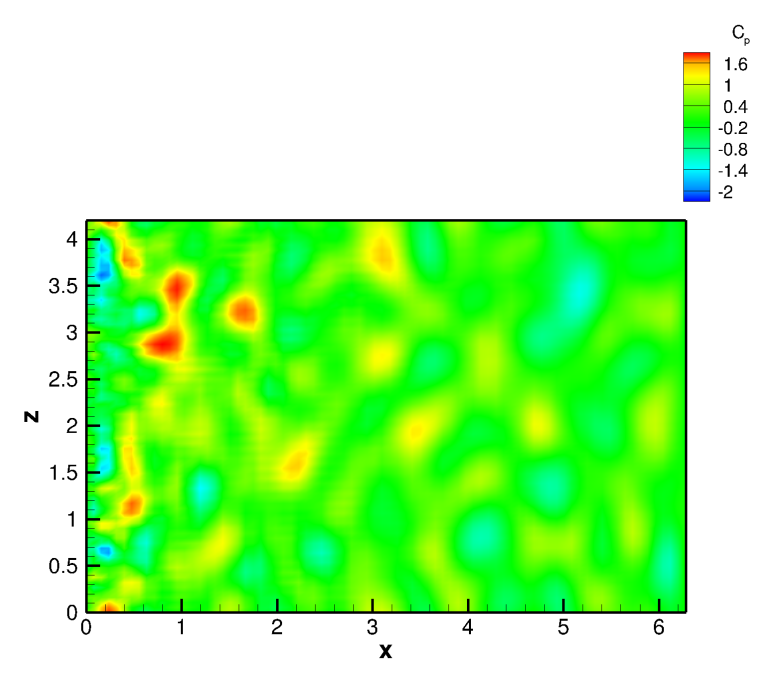

(a) Instantaneous $C_{p}$
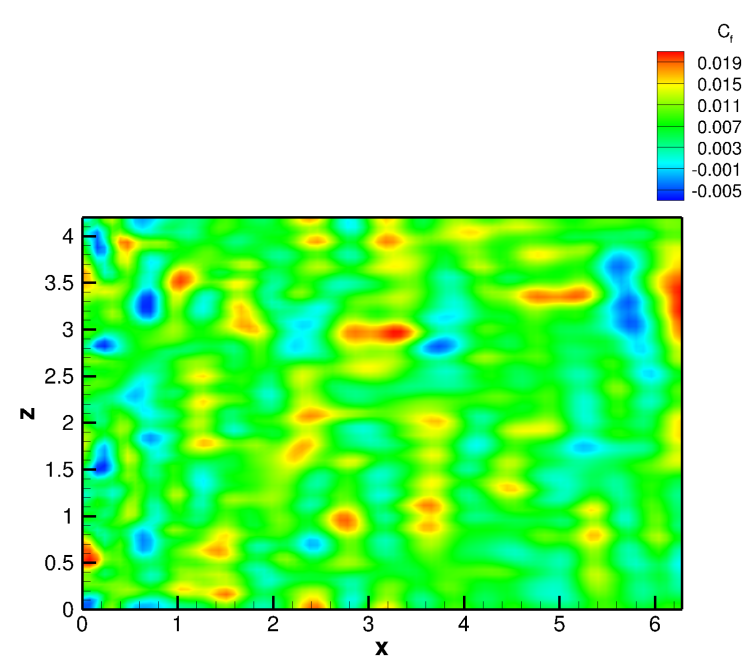

(b) Instantaneous $C_{f}$

Figure 9. Instantaneous $C_{p}$ and $C_{f}$ at all spanswise grid planes along bottom wall.

The unusually large instantaneous fluctuations of $C_{f}$ and $C_{p}$ are probably due to the propagation of acoustic waves from the inflow boundary into the domain and from the periodic boundaries in the spanwise direction. Similar fluctuations have also been observed in channel flow simulations by Park et.al. ${ }^{25}$ The presence of these waves is a matter of concern and possibly could be mitigated by the usage of advanced inflow turbulence generators coupled with nonreflective boundary conditions at the inflow for compressible flow simulations.

\section{VII.B. Results using Strelets-DES}

The Strelets DES model described in Section III was implemented in NASA's FUN3D code and results for the channel flow case are presented in this section. The simulation was carried out on the tetrahedral mesh described in section V.A. Figure 10 shows the spatial and temporal-averaged mean streamwise velocity and resolved shear stress profiles. The mean streamwise velocity profile matches the DNS data of Kim et al. ${ }^{16}$ until a $y^{+}=20$ and then the deviates sharply from the log-law resulting in what is called the classical log law defect. The maximum value of $u^{+}$at the center of the channel reaches 20 and is much larger than the DNS value of 18. The log-law mismatch also results in poor prediction of the resolved shear stress particularly towards the center of channel. Many hybrid and DES methods suffer from this pitfall.

\section{VII.C. Results using Model Invariant Hybrid RANS-LES(MI)}

The model invariant hybrid RANS-LES formulation described in Section III was implemented in FUN3D with the continuous blending of the blending parameter $\lambda$ without the source terms. The location of the RANS region in the present work was fixed at a distance of $0.1 \mathrm{~h}$ from the wall, while the location of the LES region was fixed at $0.2 h$ from the wall. The blending parameter $\lambda$ is 0 in the RANS region and 1 in the LES region, while in the region between the RANS and LES regions $\lambda$ is varied using a clamped cubic spline. The hybrid computations were carried out on the tetrahedral mesh described in section V.A. Figure 11 shows the spatial and temporal-averaged mean streamwise velocity and resolved shear stress profiles. The mean streamwise velocity is in good agreement with the DNS data of Kim et al. ${ }^{16}$ and is devoid of 


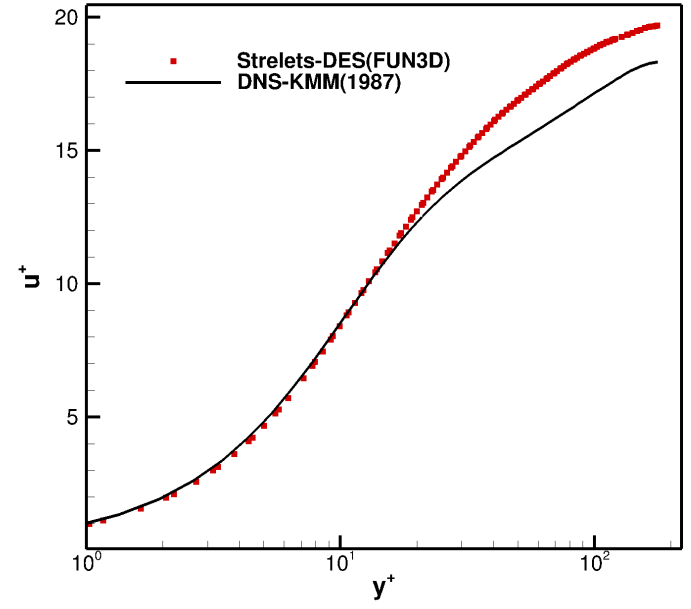

(a) Mean streamwise velocity $\langle u\rangle$

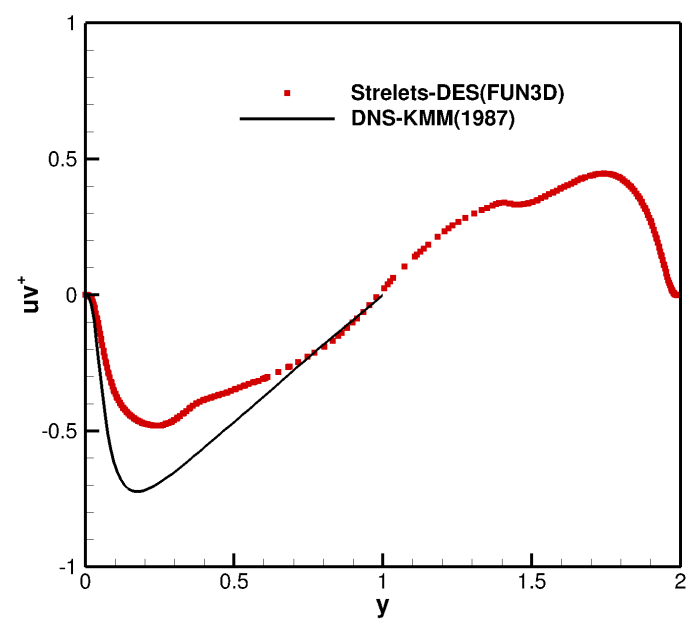

(b) Resolved shear stress $\langle u v\rangle$

Figure 10. Strelets-DES results.

the law-law mismatch. The resolved shear stress profile is in reasonable agreement with DNS results and though a small plateau is observed in the profile. Woodruff ${ }^{9}$ showed that incorporating the source terms in the model invariant approach was critical in mitigating the log-law defect and obtaining an accurate shear stress. However, in the present simulation, setting the transition zone very close to the wall permitted reasonable results to be obtained without the source terms.

For the Strelets DES and the MI methodology, both of which are based on the $k-\omega$ model, 350 modes, a length scale of $0.1 \mathrm{~h}$ and a turbulent time scale of $10 \Delta t$ as input to the isotropic turbulence inflow generator. The underlying reason being that the default freestream eddy viscosity of the $k-\omega$ model in FUN3D is quite large compared to the SA-DES model and hence a different combination of input paramenters to the turbulence generator was necessary to trigger suitable fluctuations.

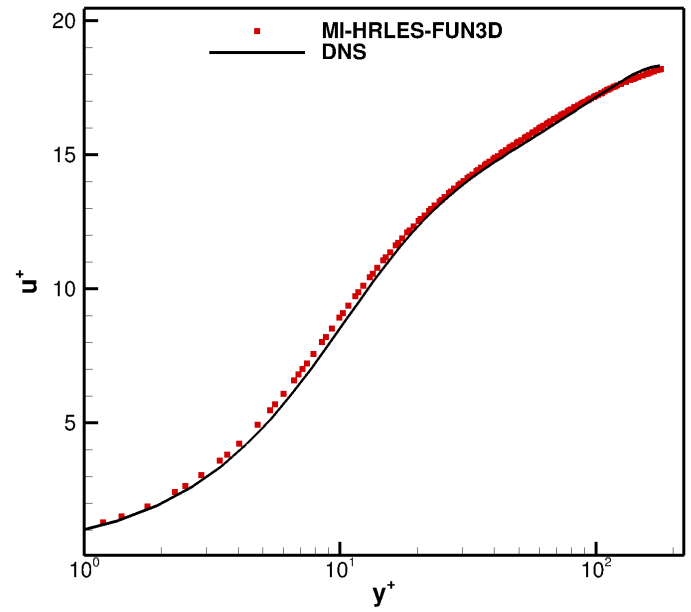

(a) Mean streamwise velocity $\langle u\rangle$

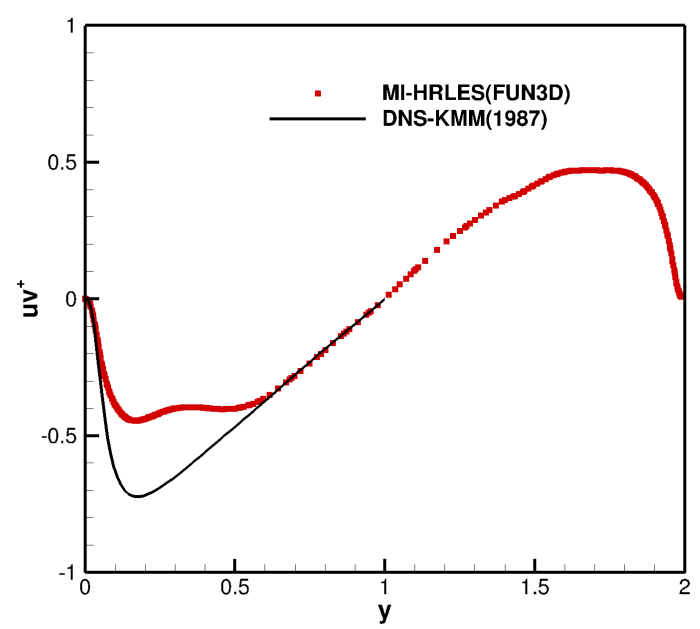

(b) Resolved shear stress $\langle u v\rangle$

Figure 11. MI results on tetrahedral mesh. 


\section{Conlcusion}

DES results using NASA's FUN3D code for the channel flow with the Spalart-Allmaras ${ }^{1}$ model were presented. The Strelets ${ }^{11}$ DES model was implemented in FUN3D, and the results were discussed. The SADES and Strelets ${ }^{11}$ DES approaches coupled with the isotropic inflow turbulence generation were presented, to provide the baseline for hybrid computations with unstructured grids. The log-law mismatch in the mean streamwise velocity with the Strelets DES was clearly observed. The model invariant hybrid RANS-LES(MI) method was implemented without the source terms and preliminary results for the hybrid computations were presented. The MI approach coupled with inflow turbulence generation was found to mitigate the classical log-layer mismatch, and provide accurate prediction of the mean flow and resolved stress. Future work would involve implementation of an advanced version of the MI method with the incorporation of source terms arising from the variation of the blending parameter, into the governing equations, adopting a better methodology to generate unstructured tetrahedral meshes for hybrid computations, grid refinement studies, and further investigation into aspects such as the sensitivity to the location of the RANS and LES zones. 


\section{References}

${ }^{1}$ Spalart, P., Jou, W., Strelets, M., and Allmaras, S., "Comments of Feasibility of LES for Wings, and on a Hybrid RANS/LES Approach," International Conference on DNS/LES, Aug. 4-8, 1997, Ruston, Louisiana., 1997.

${ }^{2}$ Nikitin, N. V., Nicoud, F., Wasistho, B., Squires, K. D., and Spalart, P. R., "An Approach to Wall Modeling in Large-Eddy Simulations," Physics of Fluids, Vol. 12, No. 7, 2000, pp. 1629-1632.

${ }^{3}$ Kawai, S. and Larsson, J., "Wall-Modeling in Large Eddy Simulation: Length Scales, Grid Resolution, and Accuracy," Physics of Fluids, Vol. 24, No. 1, 2012, pp. 015105.

${ }^{4}$ Keating, A., De Prisco, G., and Piomelli, U., "Interface Conditions for Hybrid RANS/LES Calculations," International journal of heat and fluid flow, Vol. 27, No. 5, 2006, pp. 777-788.

${ }^{5}$ Shur, M. L., Spalart, P. R., Strelets, M. K., and Travin, A. K., "Synthetic Turbulence Generators for RANS-LES Interfaces in Zonal Simulations of Aerodynamic and Aeroacoustic Problems," Flow, Turbulence and Combustion, Vol. 93, No. 1, 2014, pp. 63-92.

${ }^{6}$ Shur, M. L., Spalart, P. R., Strelets, M. K., and Travin, A. K., "A Hybrid RANS-LES Approach with Delayed-DES and Wall-Modelled LES capabilities," International Journal of Heat and Fluid Flow, Vol. 29, No. 6, 2008, pp. 1638-1649.

${ }^{7}$ Wallin, S. and Girimaji, S., "Commutation Error Mitigation in Variable-Resolution PANS Closure: Proof of Concept in Decaying Isotropic Turbulence," AIAA Paper 2011-3105, 2011.

${ }^{8}$ Woodruff, S. L., "Coupling Turbulence in Hybrid LES-RANS Techniques," Seventh International Symposium on Turbulence and Shear Flow Phenomena, Ottawa, Canada, 2010.

${ }^{9}$ Woodruff, S. L., "A New Formulation for Hybrid LES-RANS Computations," AIAA Paper 2013-2722, 2013.

${ }^{10}$ Woodruff, S., Model-Invariant Hybrid LES-RANS Computation of Separated Flow Past Periodic Hills, Springer International Publishing, Cham, 2015.

${ }^{11}$ Strelets, . M., "Detached Eddy Simulation of Massively Separated Flows," AIAA Paper 2001-879.

${ }^{12}$ Nikuradse, J., "Untersuchungen Über Die Strömungen Des Wassers in Konvergenten und Divergenten Kanälen," Forsch. Geb. Ing. Wes., Vol. 289, 1929.

${ }^{13}$ Reichardt, H., "Messungen Turbulenter Schwankungen," Naturwissenschaften., Vol. 26, No. 24/25, 1938.

${ }^{14}$ Laufer, J., "Investigation of Turbulent Flow in a Two-Dimensional Channel," NACA Report 1053, 1951.

${ }^{15}$ Compte-Bellot, G., "Contribution a L'etude De La Turbulence De Conduite," Docteur-es-Sciences thesis, Grenoble, 1963.

${ }^{16} \mathrm{Kim}$, J., Moin, P., and Moser, R., "Turbulence Statistics in Fully Developed Channel Flow at Low Reynolds Number," Journal of Fluid Mechanics, Vol. 177, 1987, pp. 133-166.

${ }^{17}$ Moin, P. and Kim, J., "Numerical Investigation of Turbulent Channel Flow," Journal of Fluid Mechanics, Vol. 118, 1982, pp. 341-377.

${ }^{18}$ Menter, F. R., "Two-Equation Eddy-Viscosity Turbulence Models for Engineering Applications," AIAA Journal, Vol. 32 , No. 8, 1994, pp. 1598-1605.

${ }^{19}$ Speziale, C., "Turbulence Modeling for Time-Dependent RANS and VLES: A Review," AIAA Journal, Vol. 36, No. 2, 1998, pp. 173-184.

${ }^{20}$ Anderson, W. K. and Bonhaus, D. L., "An Implicit Upwind Algorithm for Computing Turbulent Flows on Unstructured Grids," Comput. Fluids, Vol. 23, No. 1, 1994, pp. 1-21.

${ }^{21}$ Anderson, W., Rausch, R. D., and Bonhaus, D. L., "Implicit/Multigrid Algorithms for Incompressible Turbulent Flows on Unstructured Grids," Journal of Computational Physics, Vol. 128, No. 2, 1996, pp. 391-408.

${ }^{22}$ Biedron, R. T., Carlson, J. R., Joseph, M. D., Gnoffo, P. A., Hammond, D. P., Jones, W. T., Kleb, B., Lee-Rausch, E. M., Nielsen, E. J., Park, M. A., Rumsey, C. L., Thomas, J. L., and Wood, W. A., "FUN3D Manual: 13.3," Vol. NASA/TM2018-219808, 2016.

${ }^{23}$ Nishikawa, H. and Liu, Y., "Third-Order Edge-Based Scheme for Unsteady Problems," AIAA Paper accepted at Aviation 2018, Atlanta, Georgia, 2018.

${ }^{24}$ Davidson, L., "Using Isotropic Synthetic Fluctuations as Inlet Boundary Conditions for Unsteady Simulations," Advances and Applications in Fluid Mechanics, Vol. 1, No. 1, 2007, pp. 1-35.

${ }^{25}$ Park, G. I. and Moin, P., "Space-Time Characteristics of Wall-Pressure and Wall Shear-Stress Fluctuations in WallModeled Large Eddy Simulation," Phys. Rev. Fluids, Vol. 1, Jun 2016, pp. 024404. 